\title{
Synchronization and Stability of Elasticity Coupling Two Homodromy Rotors in a Vibration System
}

\author{
Yongjun Hou, Mingjun Du, Pan Fang, Yuwen Wang, and Liping Zhang \\ School of Mechanical Engineering, Southwest Petroleum University, Chengdu 610500, China \\ Correspondence should be addressed to Yongjun Hou; yongjunhou@126.com
}

Received 8 March 2016; Revised 24 June 2016; Accepted 4 July 2016

Academic Editor: Salvatore Strano

Copyright (C) 2016 Yongjun Hou et al. This is an open access article distributed under the Creative Commons Attribution License, which permits unrestricted use, distribution, and reproduction in any medium, provided the original work is properly cited.

\begin{abstract}
The mechanical model of an elasticity coupling 1-DOF system is proposed to implement synchronization; the simplified model is composed of a rigid body, two induction motors, and a connecting spring. Based on the Lagrange equations, the dynamic equation of the system is established. Moreover, a typical analysis method, the Poincare method, is applied to study the synchronization characteristics, and the balanced equations and stability criterion of the system are obtained. Obviously, it can be seen that many parameters affect the synchronous state of the system, especially the stiffness of the support spring, the stiffness of the connecting spring, and the installation location of the motors. Meanwhile, choose a suitable stiffness of the connecting spring $(k)$, which would play a significant role in engineering. Finally, computer simulations are used to verify the correctness of the theoretical analysis.
\end{abstract}

\section{Introduction}

The synchronization phenomenon is often encountered in nature, which is considered as an adjustment of rhythms of oscillating objects due to their internal weak couplings [1]. For example, fireflies in flocks give off flashes of light with the same frequency on a summer night; the synchronous motion of space is established between the moon's orbital motion and its rotational motion; in order to achieve selfsynchronization in the asynchronous communication techniques between the transmitter and the receiver, the two sides must simultaneously send and receive information over the same frequency and so forth. A general definition of synchronization is given in [2], and Staden and Hattingh proposed set-up describes synchronization of interconnected systems with respect to a set of functionals and captures peculiarities of both self-synchronization and controlled synchronization [3]. However, among so many engineering applications of self-synchronization, the dynamics of coupled pendulums and rotors, the synchronization of mechanical rotors, and the synchronization of modeling of nonlinearity dynamics are the best representatives. For the dynamics of coupled pendulums and rotors, Dutch scholar Huygens firstly reported the synchronization phenomenon in 1665 , two pendulum clocks hanging the common base, and the clocks exhibit synchronized motion in a short while [4]. Subsequently, Koluda et al. described the phenomenon of synchronization of clocks hanging on a common movable beam with the energy balance method and discovered how the energy is transferred between the pendula via the oscillating beam [5-7]. For the synchronization of mechanical rotors, Blekhman, the scholar of the former Soviet Union, proposed the Poincare method for the synchronization characteristics and synchronization theory of the vibrating machines [1]. Sperling et al. explored the synchronization issues of the two-plane rigid-rotor autobalancing device with numerical simulation results [8]. Balthazar et al. analyzed the self-synchronization problem of nonideal exciters by means of numerical simulations $[9,10]$. Bangchun et al. developed the average method to study the synchronization of multiple unbalanced rotors [11]. Zhao et al. proposed the average method of modified small parameters to explore the synchronization characteristics of multiple unbalanced rotors, and the process of solving the dynamic response is reduced greatly [12-14]. For the synchronization of modeling of nonlinearity dynamics, the dynamics and synchronization of coupled electromechanical systems with both cubic and quintic nonlinearities are analyzed by Ngueuteu et al. [15]. 
The model of 1-DOF pendulums coupled with a weak spring, "The Coupled Pendulums of the Kumamoto University," is proposed and Speed Gradient Energy method proposed by Kumon et al. adopted designing the controller to control the small input [16]. Fradkov and Andrievsky have put forward the harmonic linearization technique applied to studying of phase relations between coupled oscillators [17].

However, the synchronization of the model of 1-DOF pendulums coupled with a weak spring is little investigated. Therefore, this paper gives a further exploration of the coupling characteristics with the Poincare method. Finally, computer simulations are preformed to verify the results of the theoretical computation.

The structure of the paper is as follows. The analysis strategy and considered model are described in Section 2. In Section 3, the balanced equations and the synchronization stability criterion are deduced. In Section 4, the results of numerical simulations and the computer simulations are presented, which validates correctness of the theoretical computations. Also, the existence of the connecting spring has a number of potential applications in engineering. Finally, the conclusions are given in Section 5.

\section{Strategy and Model}

2.1. Strategy. The dynamic equations of a generating rotation system will be as follows [18]:

$$
\begin{aligned}
J_{i} \ddot{\varphi}_{i} & =\mu \varphi\left(\varphi_{i}, \dot{\varphi}_{i}, \ddot{\varphi}_{i}, x, \dot{x}, \ddot{x}\right), \quad(i=1,2, \ldots, k), \\
\ddot{x}+ & 2 \omega_{x} \xi_{x} \dot{x}+\omega_{x}^{2} x \\
& =\sum_{i=1}^{k} F_{i}\left(\omega t, \alpha_{1}, \ldots, \alpha_{k}\right)+\sum_{i=1}^{k} \mu F_{i+1}\left(\omega t, \alpha_{1}, \ldots, \alpha_{k}\right),
\end{aligned}
$$

where

$$
\mu \varphi_{i}=M_{e i}(\dot{\varphi})-R_{e i}(\dot{\varphi})-f_{i}\left(\varphi_{i}, \dot{\varphi}_{i}, \ddot{\varphi}_{i}, x, \dot{x}, \ddot{x}\right) .
$$

$\mu$ introduced the small parameter. $J_{i}$ is the rotational inertia of $i$ th induction motors, $M_{e i}$ is the driving torque of $i$ th induction motors, and $R_{e i}$ is the mechanical damping torque of $i$ th induction motors. $\xi_{x}$ and $\omega_{x}$ are the damping coefficient and the natural frequency of the system in $x$-directions, $\varphi_{i}, \dot{\varphi}_{i}, \ddot{\varphi}_{i}$ are the phase angle, mechanical rotational speed, and the angular acceleration of the ith induction motors, respectively. $x, \dot{x}, \ddot{x}$ are the displacement, the speed, and the average acceleration of the rigid vibrobody.

According to [1] and (1a) and (1b), the rotor synchronous behavior of vibration system would be analyzed as the following:

(1) In the synchronous state, the velocity of the rotors is assumed as $\omega$. Steady forced vibrations with $T=2 \pi / \omega$ are determined by (i.e., (1b) is considering $\mu=0$ ):

$$
x=x\left(\omega t, \alpha_{1}, \ldots, \alpha_{k}\right) .
$$

Assuming the rotors are uniformly rotating with initial phase $\alpha_{1}, \ldots, \alpha_{k}$, then the phase angle of rotors should satisfy the synchronous solutions according to the second formula (3):

$$
\varphi_{i}=\left(\omega t+\alpha_{i}\right)
$$

(2) above-mentioned basic equation may accord with such values of constants $\alpha_{1}, \ldots, \alpha_{k}$, which satisfy

$$
P_{i}\left(\alpha_{1}, \ldots, \alpha_{k}\right)=\left\langle\mu \varphi_{i}\left(\varphi_{i}, \dot{\varphi}_{i}, \ddot{\varphi}_{i}, x, \dot{x}, \ddot{x}\right)\right\rangle=0 .
$$

Here, the angle bracket $\langle *\rangle$ shows the average value for one period by the variable $t$, and symbol $*$ represents a function related to time $t$ :

$$
\langle *\rangle=\frac{1}{T} \int_{0}^{T} * d t
$$

(3) If a certain set of constants $\alpha_{1}, \ldots, \alpha_{k}$ are satisfied (5), all the roots $\chi$ of the algebraic equation,

$$
\begin{array}{cccc}
\frac{\partial\left(P_{1}-P_{k}\right)}{\partial \alpha_{1}}-\chi & \frac{\partial\left(P_{1}-P_{k}\right)}{\partial \alpha_{2}} & \cdots & \frac{\partial\left(P_{1}-P_{k}\right)}{\partial \alpha_{k-1}} \\
\frac{\partial\left(P_{2}-P_{k}\right)}{\partial \alpha_{1}} & \frac{\partial\left(P_{2}-P_{k}\right)}{\partial \alpha_{2}}-\chi & \cdots & \frac{\partial\left(P_{2}-P_{k}\right)}{\partial \alpha_{k-1}} \\
\vdots & \vdots & \vdots & \vdots \\
\frac{\partial\left(P_{k-1}-P_{k}\right)}{\partial \alpha_{1}} & \frac{\partial\left(P_{k-1}-P_{k}\right)}{\partial \alpha_{2}} & \cdots & \frac{\partial\left(P_{k-1}-P_{k}\right)}{\partial \alpha_{k-1}}-\chi
\end{array} \mid
$$

would have negative real parts; then a unique constants value $\alpha_{1}, \alpha_{2}, \ldots, \alpha_{k}$ is determined when the parameter $\mu$ is sufficiently small. Meanwhile there exists asymptotic periodic solution of (1a) and (1b). The corresponding solution is unstable only when a single root has a positive real part. For the zero or imaginary roots, an additional analysis would further be explored [1].

2.2. Model. The model of the coupling unit is shown in Figure 1 in practical industrial application. Two motors rotate in same direction connected with the coupling unit, which consists of a connecting rod, chutes, coupling springs, and slide blocks. The chute, linked to the ends of the connecting rod by welding, should be mutually paralleled. The slide block and the coupling springs are installed in the chutes. Besides, the stiffness of the connecting rod is bigger than the coupling spring and the connecting rod with smaller density.

Figure 2 describes the dynamics model of the vibration system, which mainly consists of a rigid vibrobody $m_{0}[\mathrm{~kg}]$, two motors $m_{i}[\mathrm{~kg}$ ] (for $i=1,2$ ), and the coupling unit. The two unbalanced rotors are driven by two induction motors. The vibratory body is connected with an elastic foundation through some stronger stiffness springs $k_{x}, k_{y}, k_{\psi}[\mathrm{N} / \mathrm{m}]$ in $x$-, $y$-, $\psi$-directions, which have negative effects on the movement with damping constant $C_{x}, C_{y}, C_{\psi}[\mathrm{N} \cdot \mathrm{s} / \mathrm{m}]$. Considering the unbalanced rotor as a point mass, the distance between the rotor and its own spin axes $\left(o_{i}\right)$ is denoted by $r_{i}$ $[\mathrm{m}]$. The coupling unit is simplified as a linear spring $k[\mathrm{~N} / \mathrm{m}]$, 


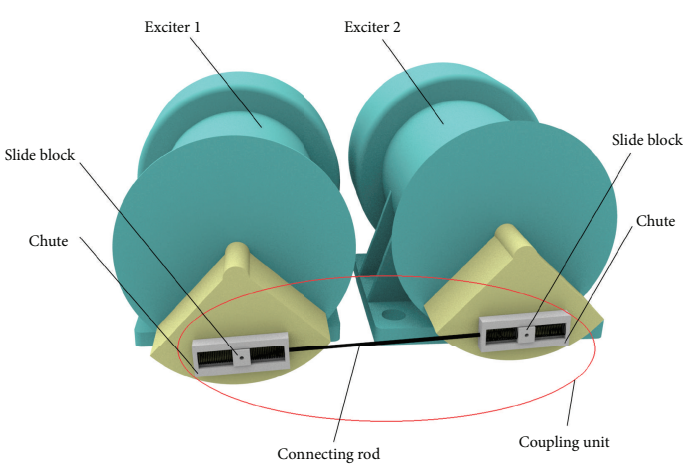

FIGURE 1: The model of the coupling unit.

which is installed in the same location of the two rotors, and the distance between connection point and the spin axes $\left(o_{i}\right)$ of the rotors is assumed as $a[\mathrm{~m}]$. The mass-center of the rigid frame is the point $\left(o^{\prime}\right)$, as illustrated in Figure 2(b), and three-reference coordinate system of the vibration system is designed as follows: the nonrotating moving frame $o^{\prime} x^{\prime} y^{\prime}$ is always parallel to the fixed coordinate frame oxy in $x$-and $y$-directions, and the moving frame $o^{\prime} x^{\prime \prime} y^{\prime \prime}$ swings around the point $\left(o^{\prime}\right)$. The three-reference system of the vibrobodies separately coincides with each other when the system is in the static equilibrium state.

Since the rigid vibrobody is supported by the elastic foundations, the vibration system exhibits three degrees of freedom; the responses $x, y$ and the angular rotation $\psi$ of the vibrating body are considered as independent coordinates. The installation angle of $i$ th motor is expressed by $\beta_{i}\left[{ }^{\circ}\right]$, and the exciters also rotate around its own spin axes, which are denoted by $\varphi_{i}[\mathrm{rad}]$. The exciters are steadily operating to overcome the ambient damp constant $C_{i}[\mathrm{~N} \cdot \mathrm{s} / \mathrm{m}]$ (for $i=$ $1,2) ; T_{m i}, T_{f i}(i=1,2)$ are the electromagnetism torque and the load torque of $i$ th exciter, respectively. For the calculation to be greatly simplified, we assume $M[\mathrm{~kg}]$ is the total mass of the vibration system and $J\left[\mathrm{~kg} \cdot \mathrm{m}^{2}\right]$ is the general inertia, $M=m_{0}+\sum_{i=1}^{2} m_{i}$.

The expressions for the kinetic energy of the system can be expressed as the following:

$$
\begin{aligned}
T & =\frac{1}{2} m_{0}\left\{\left[\dot{x}-\ell_{0} \dot{\psi} \sin \left(\beta_{0}+\psi+\pi\right)\right]^{2}+[\dot{y}\right. \\
& \left.\left.+\ell_{0} \dot{\psi} \cos \left(\beta_{0}+\psi+\pi\right)\right]^{2}\right\}+\frac{1}{2} \\
& \cdot \sum_{i=1}^{2} m_{i}\left\{\left[\dot{x}-\ell_{i} \dot{\psi} \sin \left(\beta_{i}+\psi\right)+r_{i} \dot{\varphi}_{i} \sin \varphi_{i}\right]^{2}\right. \\
& \left.+\left[\dot{y}+\ell_{i} \dot{\psi} \cos \left(\beta_{i}+\psi\right)+r_{i} \dot{\varphi}_{i} \cos \varphi_{i}\right]^{2}\right\}+\frac{1}{2} J_{0} \dot{\psi}^{2}+\frac{1}{2} \\
& \cdot \sum_{i=1}^{3} J_{i} \dot{\varphi}_{i}^{2} .
\end{aligned}
$$

Besides, considering the distance of the co-rotating induction motors is $r[\mathrm{~m}]$, and assuming the ration $(a / r \ll$
1) is infinitesimally small, so, the elongation of the coupled spring can be obtained:

$$
\Delta \ell=\ell-\ell_{0}=a\left(\cos \varphi_{1}-\cos \varphi_{2}\right) .
$$

And the potential energy of the system can be written in the following form:

$$
V=\frac{1}{2} k_{x} x^{2}+\frac{1}{2} k_{y} y^{2}+\frac{1}{2} k_{\psi} \psi^{2}+\frac{1}{2} k \Delta \ell^{2} .
$$

In addition, the viscous dissipation function of the vibration system can be computed as

$$
D=\frac{1}{2} C_{x} \dot{x}^{2}+\frac{1}{2} C_{y} \dot{y}^{2}+\frac{1}{2} C_{\psi} \dot{\psi}^{2}+\frac{1}{2} C_{1} \dot{\varphi}_{1}^{2}+\frac{1}{2} C_{2} \dot{\varphi}_{2}^{2} .
$$

The dynamics equation of the system can be obtained by the application of the Lagrange's equations:

$$
\frac{d}{d t} \frac{\partial(T-V)}{\partial \dot{q}_{i}}-\frac{\partial(T-V)}{\partial q_{i}}+\frac{\partial D}{\partial q_{i}}=Q_{i} .
$$

If $q=\left[x, y, \psi, \varphi_{1}, \varphi_{2}, \varphi_{3}\right]^{T}$ is chosen as the generalized coordinates, the generalized forces are $Q_{x}=Q_{y}=Q_{\psi}=0$ and $Q_{\varphi i}=T_{m i}-T_{f i}$. It can be seen that $m_{i} \ll m_{0}$ and $\psi \ll 1$ in the system, and the inertia coupling from asymmetry of the system can be neglected. Considering $m_{1}=m_{2}, r_{1}=r_{2}$. the kinetic equation of the vibration system is derived as

$$
\begin{aligned}
& M \ddot{x}+C_{x} \dot{x}+k_{x} x=-\sum_{i=1}^{2} m_{i} r_{i}\left(\ddot{\varphi}_{i} \sin \varphi_{i}+\dot{\varphi}_{i}^{2} \cos \varphi_{i}\right), \\
& M \ddot{y}+C_{y} \dot{y}+k_{y} y=\sum_{i=1}^{2} m_{i} r_{i}\left(\dot{\varphi}_{i}^{2} \sin \varphi_{i}-\ddot{\varphi}_{i} \cos \varphi_{i}\right), \\
& J \ddot{\psi}+C_{\psi} \dot{\psi}+k_{\psi} \psi=\sum_{i=1}^{2} m_{i} \ell_{i} r_{i}\left[\dot{\varphi}_{i}^{2} \sin \left(\varphi_{i}+\beta_{i}+\psi\right)\right. \\
& \left.-\ddot{\varphi}_{i} \cos \left(\varphi_{i}+\beta_{i}+\psi\right)\right]-C_{1}\left(\dot{\varphi}_{1}+\dot{\psi}\right)-C_{2}\left(\dot{\varphi}_{2}\right. \\
& +\dot{\psi}), \\
& J_{o 1} \ddot{\varphi}_{1}=T_{m 1}-T_{f 1}-C_{1}\left(\dot{\varphi}_{1}+\dot{\psi}\right)-m_{1} r_{1}\left[\ddot{x} \sin \varphi_{1}\right. \\
& \left.+\ddot{y} \cos \varphi_{1}\right]+m_{1} r_{1} \ell_{1}\left[\dot{\psi}^{2} \sin \left(\varphi_{1}+\beta_{1}+\psi\right)\right. \\
& \left.-\ddot{\psi} \cos \left(\varphi_{1}+\beta_{1}+\psi\right)\right]-k a^{2}\left(\cos \varphi_{2}-\sin \varphi_{1}\right) \\
& \quad \cdot \sin \varphi_{1}, \\
& J_{o 2} \ddot{\varphi}_{2}=T_{m 2}-T_{f 2}-C_{2}\left(\dot{\varphi}_{2}+\dot{\psi}\right)-m_{2} r_{2}\left[\ddot{x} \sin \varphi_{2}\right. \\
& \left.+\ddot{y} \cos \varphi_{2}\right]+m_{2} r_{2} \ell_{2}\left[\dot{\psi}^{2} \sin \left(\varphi_{2}+\beta_{2}+\psi\right)\right. \\
& \left.\quad-\ddot{\psi} \cos \left(\varphi_{2}+\beta_{2}+\psi\right)\right]-k a^{2}\left(\cos \varphi_{1}-\sin \varphi_{2}\right) \\
& +\sin \varphi_{2} .
\end{aligned}
$$




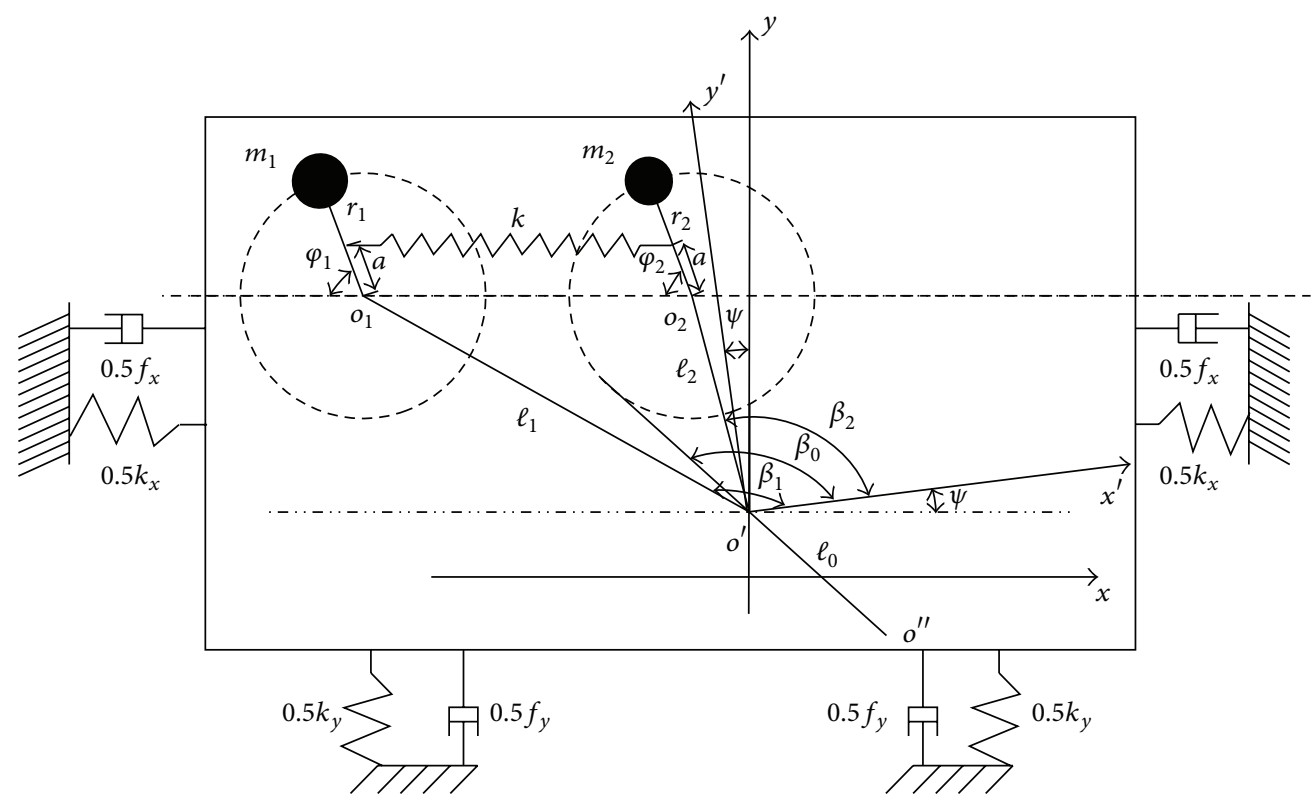

(a)

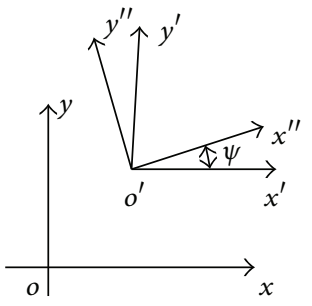

(b)

FIGURE 2: The model of the vibration system. (a) Dynamic model of the motors coupled with a weak spring and (b) the reference coordinate system.

\section{Method Description}

3.1. Coupling Characteristics. According to the Poincare method (i.e., based on the fundamental equations (1a) and (1b)), introducing the small parameter $\mu$ into (13), owing to the damping of the system being very small and the influence of the small parameter which can be ignored, then a new form of (13) is given:

$$
\begin{aligned}
& M \ddot{x}+k_{x} x=-\sum_{i=1}^{2} m_{i} r_{i}\left(\ddot{\varphi}_{i} \sin \varphi_{i}+\varphi_{i}^{2} \cos \varphi_{i}\right)-\mu f_{x}^{\prime} \dot{x}, \\
& M \ddot{y}+k_{y} y=\sum_{i=1}^{2} m_{i} r_{i}\left(\dot{\varphi}_{i}^{2} \sin \varphi_{i}-\ddot{\varphi}_{i} \cos \varphi_{i}\right)-\mu f_{y}^{\prime} \dot{y}, \\
& J \ddot{\psi}+k_{\psi} \psi=\sum_{i=1}^{2} m_{i} \ell_{i} r_{i}\left[\dot{\varphi}_{i}^{2} \sin \left(\varphi_{i}+\beta_{i}+\psi\right)\right. \\
& \left.-\ddot{\varphi}_{i} \cos \left(\varphi_{i}+\beta_{i}+\psi\right)\right]-\mu f_{\psi}^{\prime} \dot{\psi}, \\
& J_{o 1} \ddot{\varphi}_{1}=\mu \varphi_{1}\left(\varphi_{2}, \dot{\varphi}_{1}, \varphi_{1}, \ddot{\psi}, \dot{\psi}, \ddot{x}, \ddot{y}\right), \\
& J_{o 2} \ddot{\varphi}_{2}=\mu \varphi_{2}\left(\dot{\varphi}_{2}, \varphi_{2}, \ddot{\psi}, \dot{\psi}, \ddot{x}, \ddot{y}\right),
\end{aligned}
$$

where

$$
\begin{aligned}
\mu \varphi_{1} & =T_{m 1}-T_{f 1}-m_{1} r_{1}\left[\ddot{x} \sin \varphi_{1}+\ddot{y} \cos \varphi_{1}\right] \\
& +m_{1} r_{1} \ell_{1}\left[\dot{\psi}^{2} \sin \left(\varphi_{1}+\beta_{1}+\psi\right)\right. \\
& \left.-\ddot{\psi} \cos \left(\varphi_{1}+\beta_{1}+\psi\right)\right]-k a^{2}\left(\cos \varphi_{2}\right. \\
& \left.-\sin \varphi_{1}\right) \sin \varphi_{1}, \\
\mu \varphi_{2} & =T_{m 2}-T_{f 2}-m_{2} r_{2}\left[\ddot{x} \sin \varphi_{2}+\ddot{y} \cos \varphi_{2}\right] \\
& +m_{2} r_{2} \ell_{2}\left[\dot{\psi}^{2} \sin \left(\varphi_{2}+\beta_{2}+\psi\right)\right. \\
& \left.-\ddot{\psi} \cos \left(\varphi_{2}+\beta_{2}+\psi\right)\right]-k a^{2}\left(\cos \varphi_{1}\right. \\
& \left.-\sin \varphi_{2}\right) \sin \varphi_{2} .
\end{aligned}
$$

Base on [14], when the two rotors synchronously rotate $\dot{\varphi}_{i}=\omega_{m}$, the electromagnetic torque can be linearized as

$$
T_{e i}=n_{p} \frac{L_{m i}^{2} U_{S O}^{2}}{L_{s i} \omega_{m} R_{r i}}\left(\omega_{s}-n_{p} \omega_{m}\right),
$$

where $L_{m i}$ and $L_{s i}$ are the mutual inductance and stator inductance of the $i$ th induction motor, respectively; $n_{p}$ is the pole numbers of induction motor; $\omega_{m}$ is the synchronous angular velocity; $\omega_{s}$ is the power supply frequency of the grid; $R_{r i}$ is rotor resistance of the $i$ th induction motor; $U_{S O}$ is the amplitude of the stator voltage.

Here, the effect of the small parameter $(\mu)$ can be neglected. Introducing the following dimensionless parameters: the nature frequency is denoted by $\omega_{x}, \omega_{y}, \omega_{\varphi}$ in $x-, y-, \psi$-directions, respectively. The average angular velocity of the oscillating screen motor is denoted by $\omega_{m}$. The frequency ratios of the vibration system are denoted by $\eta_{x}, \eta_{y}, \eta_{\psi}:$

$$
\begin{aligned}
& r_{m}=\frac{m_{1}}{M}, \\
& r_{e}=\frac{m_{1}}{J}, \\
& \omega_{x}=\sqrt{\frac{k_{x}}{M}}, \\
& \omega_{y}=\sqrt{\frac{k_{y}}{M}}, \\
& \omega_{\psi}=\sqrt{\frac{k_{\psi}}{J}},
\end{aligned}
$$




$$
\begin{aligned}
& \eta_{x}=\frac{\omega_{m}}{\omega_{x}}, \\
& \eta_{y}=\frac{\omega_{m}}{\omega_{y}}, \\
& \eta_{\psi}=\frac{\omega_{m}}{\omega_{\psi}} .
\end{aligned}
$$

Applying the dimensionless parameters to (18), the steady responses in $x-, y$-, $\psi$-directions are obtained:

$$
\begin{aligned}
& x=\frac{r_{m} \eta_{x}^{2}}{\eta_{x}^{2}-1}\left(r_{1} \cos \varphi_{1}+r_{2} \cos \varphi_{2}\right), \\
& y=-\frac{r_{m} \eta_{y}^{2}}{\eta_{y}^{2}-1}\left(r_{1} \sin \varphi_{1}+r_{2} \sin \varphi_{2}\right), \\
& \psi=-\frac{r_{e} \eta_{\psi}^{2}}{\eta_{\psi}^{2}-1}\left(r_{1} \sin \left(\varphi_{1}+\beta_{1}\right)+r_{2} \sin \left(\varphi_{2}+\beta_{2}\right)\right) .
\end{aligned}
$$

3.2. Synchronization Criterion. Assuming $\alpha_{i}$ are the initial phase of the $i$ th unbalanced rotor, the phase angular $\left(\varphi_{i}\right)$ of the $i$ th unbalanced rotor is be defined as follows:

$$
\begin{aligned}
& \varphi_{1}=\omega t+\alpha_{1}, \\
& \varphi_{2}=\omega t+\alpha_{2} .
\end{aligned}
$$

Substituting (19) into (18), a new form is given:

$$
\begin{aligned}
& x= \frac{r_{m} \eta_{x}^{2}}{\eta_{x}^{2}-1}\left[r_{1} \cos \left(\omega t+\alpha_{1}\right)+r_{2} \cos \left(\omega t+\alpha_{2}\right)\right], \\
& y=-\frac{r_{m} \eta_{y}^{2}}{\eta_{y}^{2}-1}\left[r_{1} \sin \left(\omega t+\alpha_{1}\right)+r_{2} \sin \left(\omega t+\alpha_{2}\right)\right], \\
& \psi=-\frac{r_{e} \eta_{\psi}^{2}}{\eta_{\psi}^{2}-1}\left[r_{1} \sin \left(\omega t+\alpha_{1}+\beta_{1}\right)\right. \\
&\left.\quad+r_{2} \sin \left(\omega t+\alpha_{2}+\beta_{2}\right)\right] .
\end{aligned}
$$

The parameter $(\alpha)$ is considered as the phase difference between the two rotors; we have

$$
\alpha=\alpha_{2}-\alpha_{1}
$$

$$
\begin{aligned}
& \text { According to (5), } P_{i} \text { is expressed as } \\
& P_{1}=\left\langle\mu \varphi_{1}\right\rangle=\frac{1}{T} \int_{0}^{T}\left(\mu \varphi_{1}\right) d t \\
& =T_{m 1}-T_{f 1} \\
& -\frac{1}{2} m_{1} r_{m} r_{1} \omega^{2} r_{2}\left(\frac{\eta_{x}^{2}}{\eta_{x}^{2}-1}+\frac{\eta_{y}^{2}}{\eta_{y}^{2}-1}\right) \sin (\alpha) \\
& -\frac{1}{2} m_{1} r_{1} \ell_{1} r_{e} \omega^{2} r_{2} \frac{\eta_{\psi}^{2}}{\eta_{\psi}^{2}-1} \sin \left(\alpha+\beta_{2}-\beta_{1}\right) \\
& +\frac{1}{2} k a^{2}[\sin (\alpha)+1]=0 \text {, } \\
& P_{2}=\left\langle\mu \varphi_{2}\right\rangle=\frac{1}{T} \int_{0}^{T}\left(\mu \varphi_{2}\right) d t \\
& =T_{m 2}-T_{f 2} \\
& +\frac{1}{2} m_{2} r_{m} r_{2} \omega^{2} r_{1}\left(\frac{\eta_{x}^{2}}{\eta_{x}^{2}-1}+\frac{\eta_{y}^{2}}{\eta_{y}^{2}-1}\right) \sin (\alpha) \\
& -\frac{1}{2} m_{2} r_{2} \ell_{2} r_{e} \omega^{2} r_{1} \frac{\eta_{\psi}^{2}}{\eta_{\psi}^{2}-1} \sin \left(-\alpha+\beta_{1}-\beta_{2}\right) \\
& +\frac{1}{2} k a^{2}[-\sin (\alpha)+1]=0 .
\end{aligned}
$$

During the synchronization, the excessive torque $Z_{s}(\omega)$ of the rotors is equal to zero:

$$
Z_{s}(\omega)=T_{m i}-T_{f i}=0, \quad(i=1,2) .
$$

Therefore, the balanced equation of synchronization of the vibrating system is established:

$$
2 \mu_{1} \sin (\alpha)+\mu_{2} \sin \left(\alpha+\beta_{2}-\beta_{1}\right)-2 \mu_{3} \sin (\alpha)=0,
$$

where

$$
\begin{aligned}
& \mu_{1}=m_{1} r_{1} r_{2} r_{m} \omega^{2}\left(\frac{\eta_{x}^{2}}{\eta_{x}^{2}-1}+\frac{\eta_{y}^{2}}{\eta_{y}^{2}-1}\right), \\
& \mu_{2}=m_{1} r_{1} r_{2} r_{e}\left(\ell_{1}+\ell_{2}\right) \omega^{2} \frac{\eta_{\psi}^{2}}{\eta_{\psi}^{2}-1} \\
& \mu_{3}=k a^{2}
\end{aligned}
$$

3.3. Stability Criterion of Synchronous. According to (5) and (7), the following criterion of synchronous stability is obtained:

$$
\begin{aligned}
\chi= & \frac{\partial\left(P_{1}-P_{2}\right)}{\partial \alpha_{1}} \\
= & m_{1} r_{m} r_{1} \omega^{2} r_{2}\left(\frac{\eta_{x}^{2}}{\eta_{x}^{2}-1}+\frac{\eta_{y}^{2}}{\eta_{y}^{2}-1}\right) \cos (\alpha) \\
& +\frac{1}{2} m_{1} r_{1} r_{e} \omega^{2} r_{2} \frac{\eta_{\psi}^{2}}{\eta_{\psi}^{2}-1}\left(\ell_{1}+\ell_{2}\right) \cos \left(\alpha+\beta_{2}-\beta_{1}\right) \\
& -k a^{2} \cos (\alpha)<0 .
\end{aligned}
$$


TABLE 1: Parameter values.

\begin{tabular}{|c|c|c|c|c|}
\hline \multicolumn{4}{|c|}{ Parameter values for system equation (13) } & \multirow{2}{*}{$\begin{array}{l}\text { Parameter values according to } \\
\text { dimensionless equation (17) }\end{array}$} \\
\hline Unbalanced rotors for $i=1,2$ & Vibroplatform & Elastic spring & Other parameters & \\
\hline$m_{i}=2[\mathrm{~kg}]$ & $M=80[\mathrm{~kg}]$ & $k=0 \sim 1.4 \times 10^{5}[\mathrm{~N} / \mathrm{m}]$ & $\beta_{1}=5 \pi / 6,3 \pi / 4$ & $r_{m}=0.025$ \\
\hline$r_{i}=0.02[\mathrm{~m}]$ & $J=8\left[\mathrm{~kg} \cdot \mathrm{m}^{2}\right]$ & $a=0.01[\mathrm{~m}]$ & $\beta_{2}=46 \pi / 225, \pi / 4$ & $r_{e}=0.25$ \\
\hline$\omega_{m}=152 \sim 158[\mathrm{rad} / \mathrm{s}]$ & $k_{x}=4.0 \times 10^{4} \sim 2.0 \times 10^{8}[\mathrm{~N} / \mathrm{m}]$ & & $\ell_{1}=0.6,0.4[\mathrm{~m}]$ & $n_{x}=0.1 \sim 7$ \\
\hline \multirow[t]{5}{*}{$C_{i}=0.01[\mathrm{~N} \cdot \mathrm{s} / \mathrm{m}]$} & $k_{y}=4.0 \times 10^{4} \sim 2.0 \times 10^{8}[\mathrm{~N} / \mathrm{m}]$ & & $\ell_{2}=0.5,0.4[\mathrm{~m}]$ & $n_{y}=0.1 \sim 7$ \\
\hline & $k_{\psi}=4.0 \times 10^{3} \sim 2.0 \times 10^{7}[\mathrm{~N} / \mathrm{m}]$ & & & $n_{\varphi}=0.1 \sim 7$ \\
\hline & $f_{x}=1000[\mathrm{~N} /(\mathrm{m} / \mathrm{s})]$ & & & \\
\hline & $f_{y}=1000[\mathrm{~N} /(\mathrm{m} / \mathrm{s})]$ & & & \\
\hline & $f_{z}=1000[\mathrm{~N} /(\mathrm{m} / \mathrm{s})]$ & & & \\
\hline
\end{tabular}

So, the stability of synchronization of the system can be simplified as

$$
2 \mu_{1} \cos (\alpha)+\mu_{2} \cos \left(\alpha+\beta_{2}-\beta_{1}\right)-2 \mu_{3} \cos (\alpha)<0
$$

Only the system parameters satisfy (24) and (27); the synchronization of the vibration system can be implemented in the considered case.

\section{Numeric Verification}

Above-mentioned sections derived the balanced equations and the stability criterion of synchronization. The theoretical analysis and simulation results will be presented in this section.

4.1. Analysis of Numerical Results. It is easy to find that the balanced equations are related to the system parameters $\eta_{x}, \eta_{y}, \eta_{\varphi}, r_{m}, r_{e}, k$, and so forth, which seriously influence the stability of self-synchronization of the vibration system. The stable phase difference can be calculated by applying numerical method. Considering the system parameters $r_{m}, r_{e}$ are constant, under the condition that the balanced equations and the stability criterion of synchronization are satisfied, we further investigated the relationship between variable stiffness coefficient $(k)$ of the connecting spring, the frequency ratios $\left(\eta_{x}, \eta_{y}, \eta_{\varphi}\right)$, and the stable phase difference in the different installation positions. The parameter values corresponding to general engineering application are given in Table 1. According to (18), the dimensionless values parameters, as shown in Table 1, are obtained. Owing to the stiffness coefficients $\left(k_{x}, k_{y}, k_{\varphi}\right)$ being separately transformed into frequency ration $\left(\eta_{x}, \eta_{y}, \eta_{\psi}\right)$ in $x$-, $y$-, and $\psi$-directions. Some parameters seriously influence the stability of synchronization, including the supporting spring stiffness, the stiffness of elastic spring $k$, installation location, and so forth.

4.2. The Analysis of the Connecting Spring. It is important to select appropriate stiffness of the connecting spring in engineering. This section has analyzed that the stiffness of the connecting spring is influences on the phase difference in the synchronization state. The system parameters are shown in Table 1.
The analysis results for $n_{x}=n_{y}=n_{\varphi}=5.817, \ell_{1}=$ $0.6[\mathrm{~m}], \ell_{2}=0.5[\mathrm{~m}], \beta_{1}=5 \pi / 6$, and $\beta_{2}=46 \pi / 225$ are shown in Figure 4(a); here, the spring stiffness is $k_{x}=$ $k_{y}=58276.2[\mathrm{~N} / \mathrm{m}], k_{\varphi}=5827.6[\mathrm{~N} / \mathrm{rad}]$, and the other parameters are identical with the Table 1 . When $k=0$, the phase difference $(\alpha)$ of co-rotating motors stabilize at -1.5 [rad]. It is found that within the limits of the stiffness the phase difference is close to $0^{\circ}$ with stiffness increasing, which results in both vibration amplitude and the screening efficiency of the system which could be improved. When $k=1.001 \times 10^{5}[\mathrm{~N} / \mathrm{m}]$, the phase difference is -0.2401 [rad], which is in agreement with the results obtained for the case of theoretical solution (i.e., the stable phase difference in Figure 3(a) is equal to -0.2185 [rad]). The other analysis results for $n_{x}=n_{y}=n_{\varphi}=6.704, \ell_{1}=0.4[\mathrm{~m}]$, $\ell_{2}=0.4[\mathrm{~m}], \beta_{1}=3 \pi / 4$, and $\beta_{2}=\pi / 4$ are shown in Figure 4(b). In the considering case, the spring stiffness is $k_{x}=k_{y}=43875.4[\mathrm{~N} / \mathrm{m}]$ and $k_{\varphi}=4387.5[\mathrm{~N} / \mathrm{rad}]$, and the other parameter is consistent with Table 1 . When $k=0$, there is no stable phase difference. An unstable state of the vibration system can transform into a more stable state when the stiffness of the connecting spring is increased, and the above similar conclusions can be obtained. Comparing the theoretic calculation with Figure 3(b), the value of the stable phase difference is according to the theoretical computation (i.e., the stable phase difference in Figure 3(b) is equal to -0.1699 [rad]; here, the stable phase difference is equal to $-0.1738[\mathrm{rad}])$.

4.3. Computer Simulations. Further computer simulations have been performed to the correctness of above-mentioned theoretical analysis. We solved the dynamics equation (14) by applying the Runge-Kutta routine. Here, consider the parameters of the two motors to be identical, which are shown in Table 1.

4.3.1. For $n_{x}=n_{y}=n_{\varphi}=5.817, k=10000[\mathrm{~N} / \mathrm{m}], \ell_{1}=$ $0.6[\mathrm{~m}], \ell_{2}=0.5[\mathrm{~m}], \beta_{1}=5 \pi / 6$, and $\beta_{2}=46 \pi / 225$. Simulation results for the dimensionless parameters are shown in Figure 5. Here, the spring stiffness is $k_{x}=k_{y}=$ $58276.2[\mathrm{~N} / \mathrm{m}], k_{\varphi}=5827.6[\mathrm{~N} / \mathrm{rad}]$, and $k=10000$ $[\mathrm{N} / \mathrm{m}]$ and the other parameter is identical with Table 1. When the two induction motors are simultaneously provided 


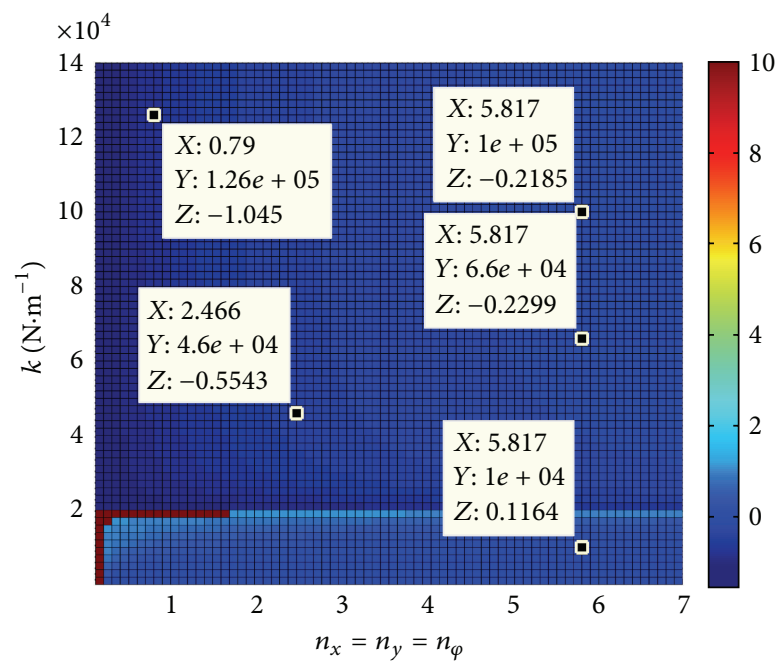

(a)

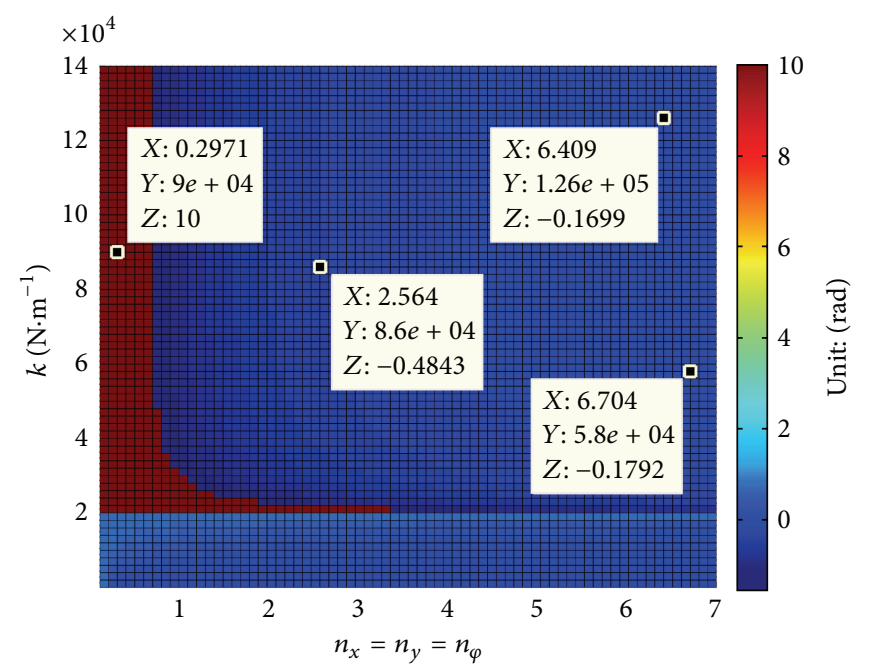

(b)

FIGURE 3: Stable phase difference with theoretical computation for (a) $\ell_{1}=0.6[\mathrm{~m}], \ell_{2}=0.5[\mathrm{~m}], \beta_{1}=5 \pi / 6$, and $\beta_{2}=46 \pi / 225 ;(\mathrm{b})$ $\ell_{1}=0.4[\mathrm{~m}], \ell_{2}=0.4[\mathrm{~m}], \beta_{1}=3 \pi / 4$, and $\beta_{2}=\pi / 4$. In this figure, coordinate $X$ represents the value of frequency ration $n_{x}=n_{y}=n_{\varphi} ;$ coordinate $Y$ represents the value of the connecting spring $k$; coordinate $Z$ represents the value of stable phase difference $\alpha$; $\alpha=10$ [rad] shows that there is no stable phase.

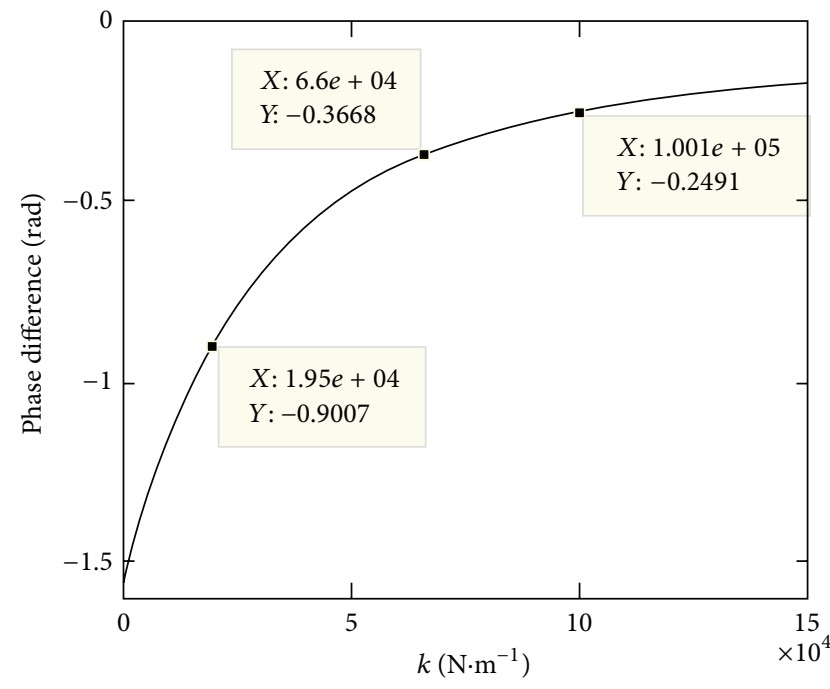

(a)

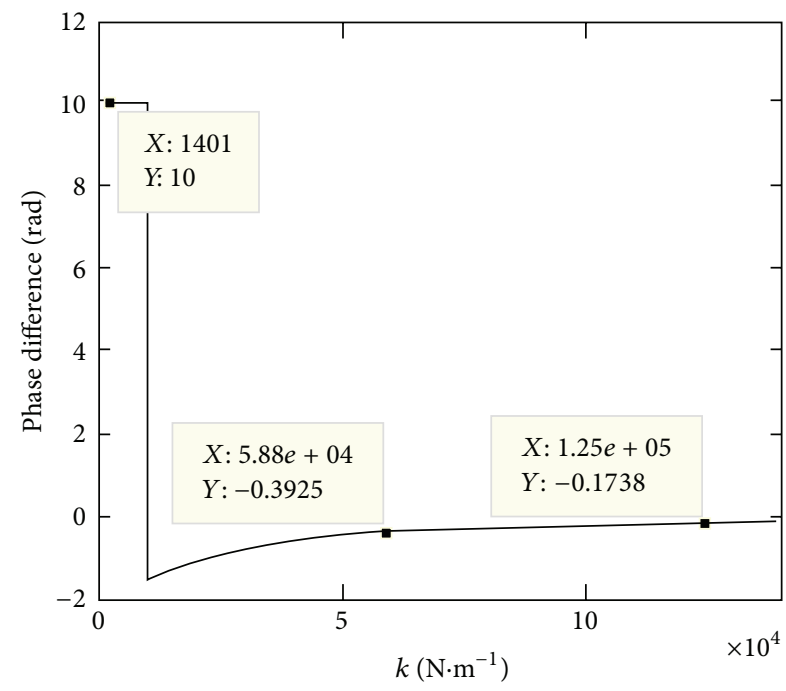

(b)

FiguRE 4: Stable phase difference is changed with the stiffness of the connecting spring for (a) $n_{x}=n_{y}=n_{\varphi}=5.817 ;$ (b) $n_{x}=n_{y}=n_{\varphi}=6.704$; In this figure, coordinate $X$ represents the stiffness of the connecting spring; coordinate $Y$ represents the phase difference ( $\alpha$ ) of co-rotating motors; $\alpha=10$ [rad] shows that there is no stable phase.

with the electromagnetic force, the angular acceleration of the two rotors is identical, as shown in Figure 5(d). At $2 \mathrm{~s}$, the synchronization process of the vibration system is implemented, and the synchronous angular velocity is 155 $[\mathrm{rad} / \mathrm{s}]$, as illustrated in Figure 5(d). The phase difference $\alpha$ is near -0.005 [rad] (in Figure 5(f)), which is coincided with the approximate value of theoretical analysis (the value of stable phase is 0.116 [rad] in Figure 3(a)). In addition, the vibrating body is excited, and the coupling torques (in Figure 5(e)), keeping the vibration system working in steady synchronization state, are approximated $1.79[\mathrm{~N} / \mathrm{m}]$, and the responses of the vibrating body are displayed in $x-, y$-, $\psi$-directions, respectively (in Figures 5(a), 5(b), and $5(\mathrm{c}))$.

4.3.2. For $n_{x}=n_{y}=n_{\varphi}=6.409, k=126000[\mathrm{~N} / \mathrm{m}], \ell_{1}=$ $\ell_{2}=0.4[\mathrm{~m}], \beta_{1}=3 \pi / 4$, and $\beta_{2}=\pi / 4$. To further verify the analysis results, it is necessary for two exciters coupled with a weak spring to give other results of computer simulation. The simulation results are shown in Figure 6; here, the spring stiffness is $k_{x}=k_{y}=48007.5[\mathrm{~N} / \mathrm{m}], k_{\varphi}=4800.8[\mathrm{~N} / \mathrm{rad}]$, and $k=126000[\mathrm{~N} / \mathrm{m}]$, and the other parameters of the 


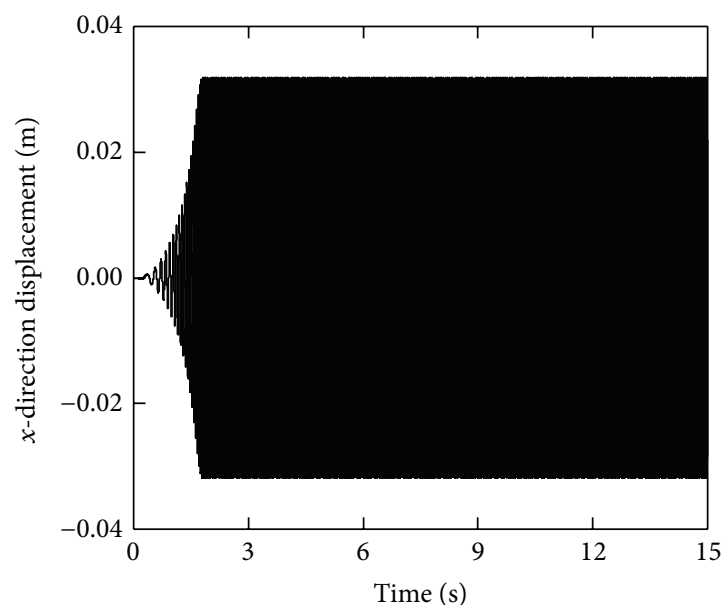

(a)

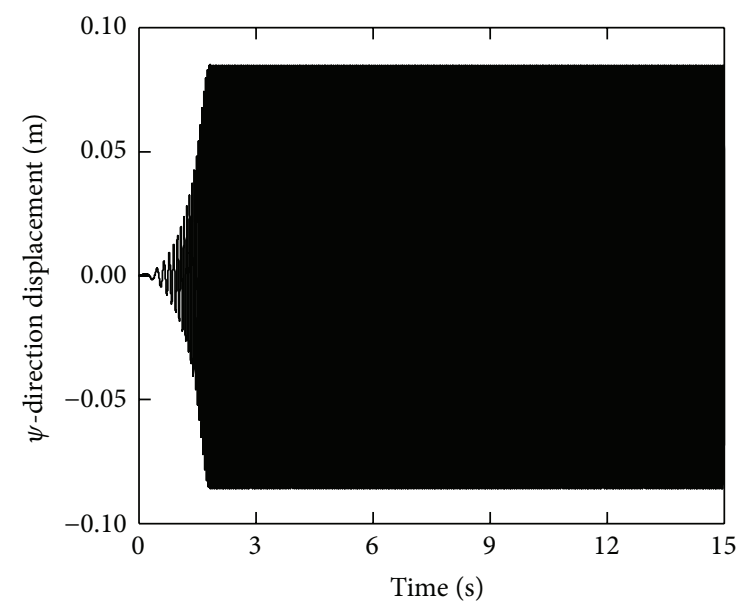

(c)

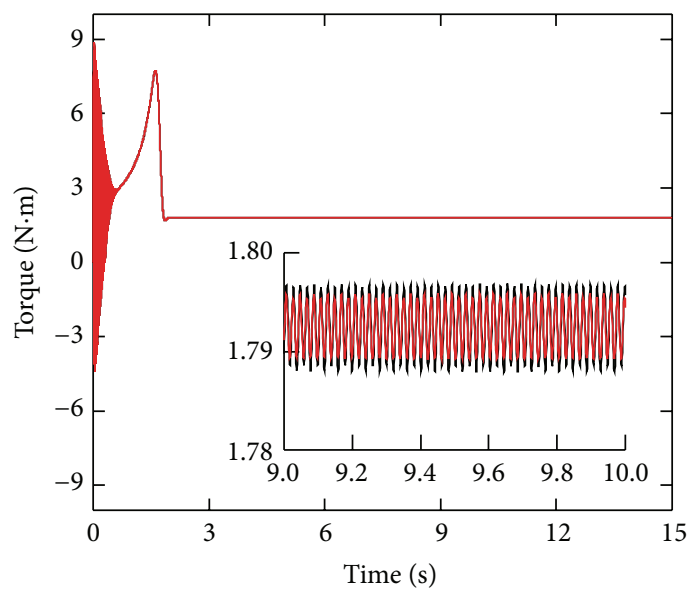

- Motor 1

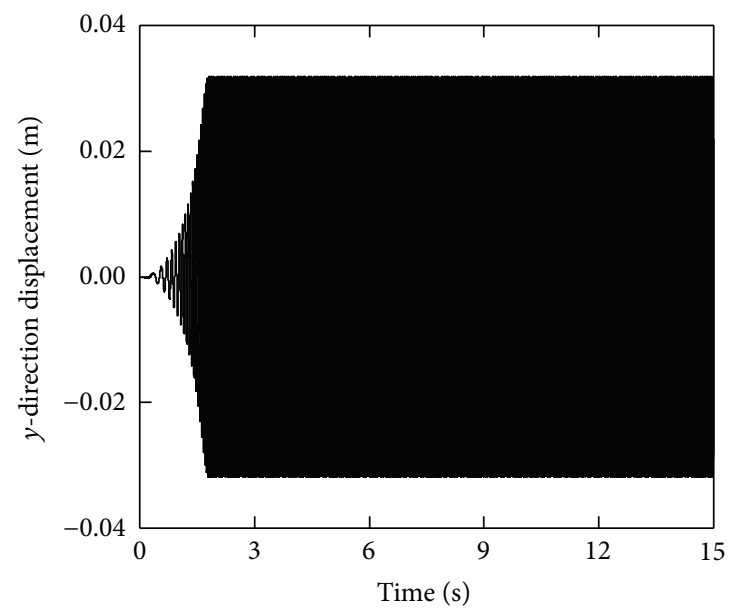

(b)

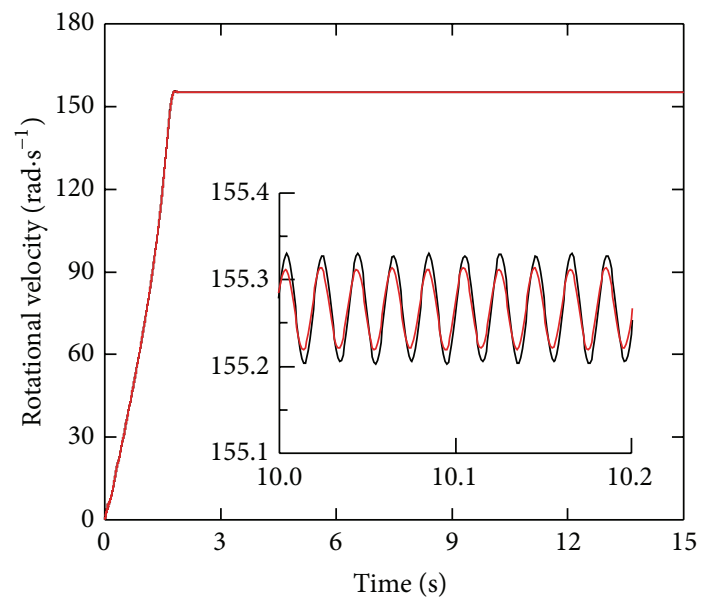

- Motor 1

- Motor 2

(d)

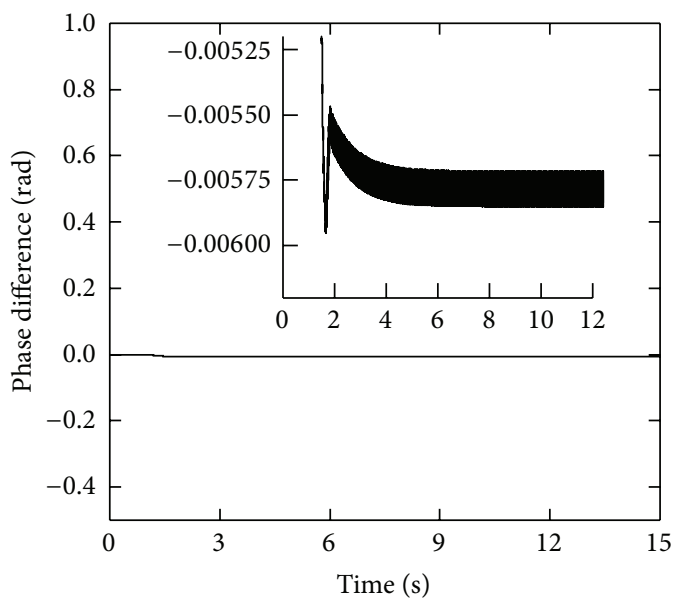

(f)

Figure 5: Results for the computer simulation: (a), (b), and (c) displacement responses of the vibrating body in $x$-, $y$-, $\psi$-directions, respectively; (d) rotational velocities of the two rotors; (e) electromagnetic torques of the two rotors; (f) phase difference of unbalanced rotors. 


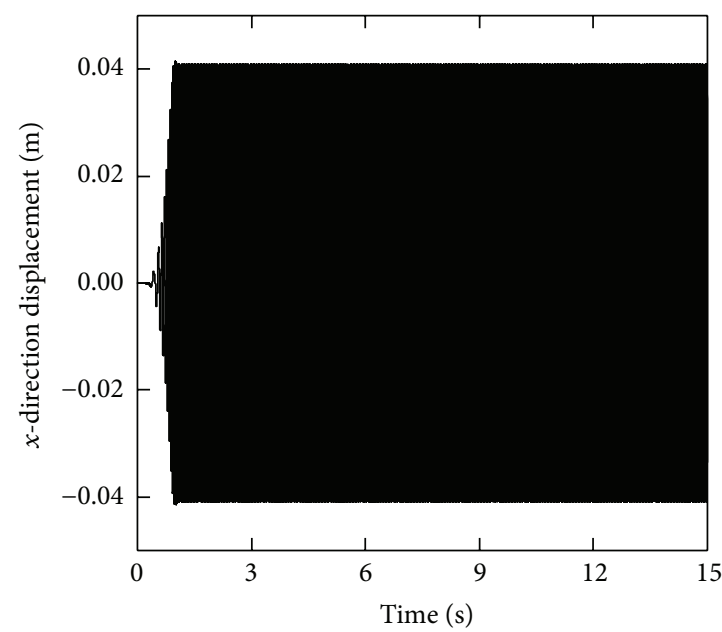

(a)

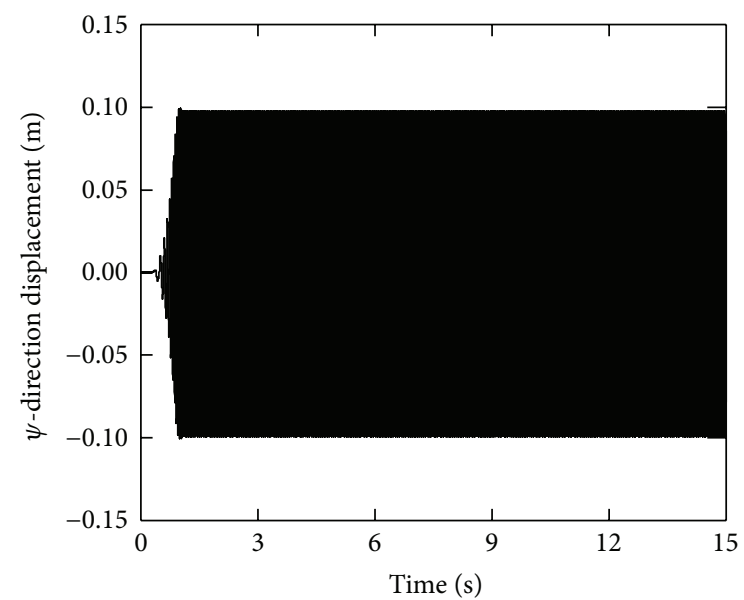

(c)

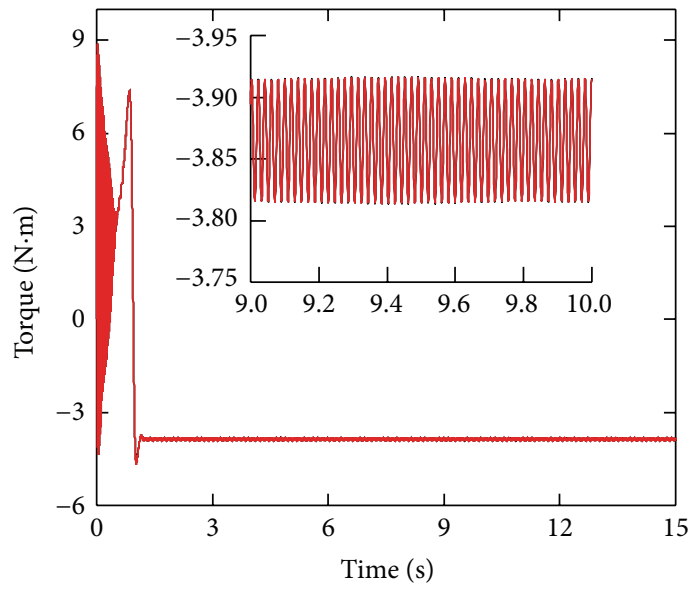

- Motor 1

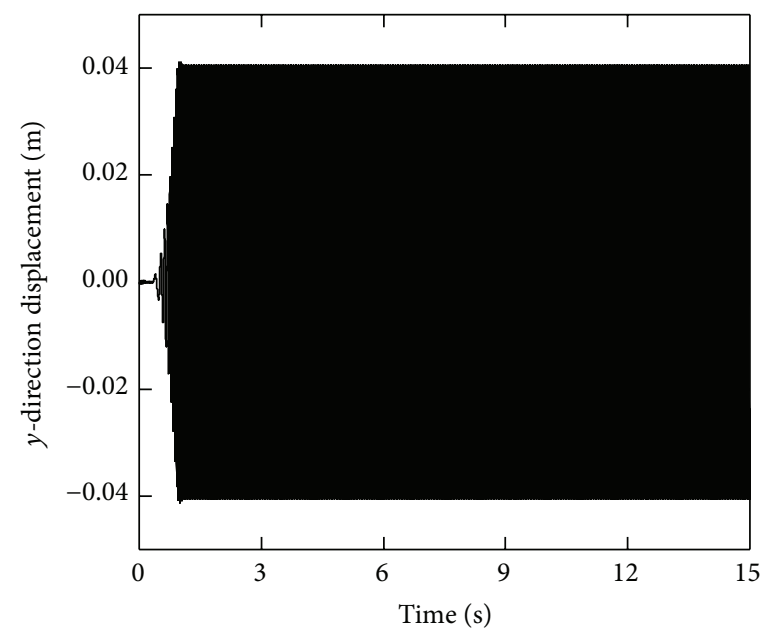

(b)

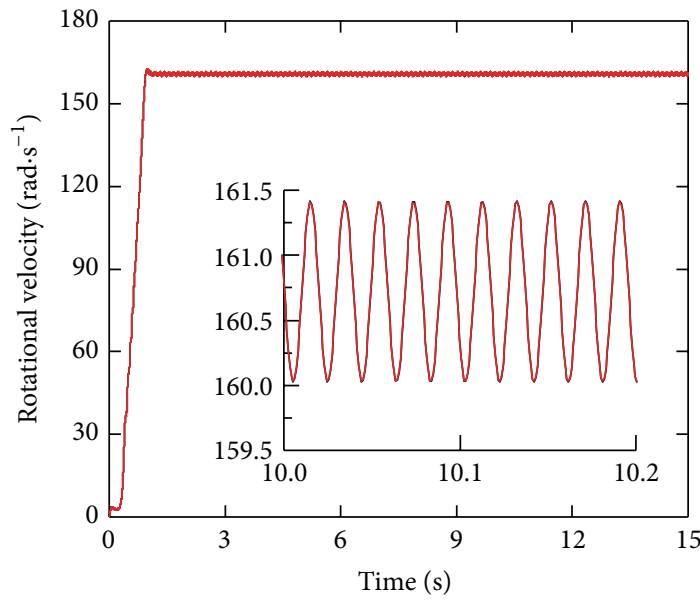

- Motor 1

- Motor 2

(d)

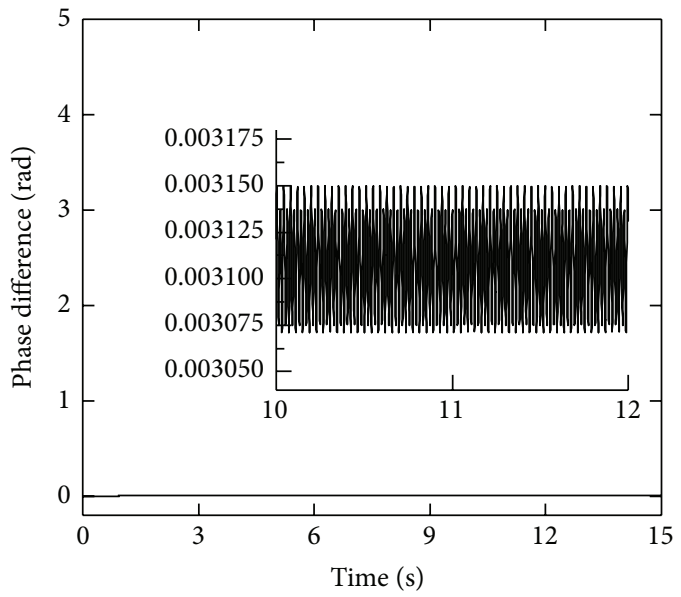

(f)

(e)

Figure 6: Results for the computer simulation: (a), (b), and (c) displacement responses of the vibrating body in $x$-, $y$-, $\psi$-directions, respectively; (d) rotational velocities of the two rotors; (e) electromagnetic torques of the two rotors; (f) phase difference of unbalanced rotors. 
system are coincided with the Table 1. During the starting process of the vibration system, owing to the rotational inertia of two rotors being identical, so the angular acceleration of two induction motors is equal (in Figure 6(d)). After about $1 \mathrm{~s}$, the synchronous state of system is implemented, and the synchronous velocity of two unbalanced rotors is 160.8 $[\mathrm{rad} / \mathrm{s}]$ (in Figure 6(d)). The phase difference of two rotors is shown in Figure 6(f), comparing numerical results with Figure 3(b); it can be seen that the computer simulation results further accord with the results of theoretical analysis. (The phase difference of Figure 6(f) stabilized at 0.003 [rad]; the stable phase difference of Figure 3(b) is equal to -0.1699 [rad].) Additionally, the electromagnetic torque is about $-3.86[\mathrm{~N} \cdot \mathrm{m}]$. The displacements of the vibrating body in $x-, y$-, and $\psi$-directions are presented in Figures 6(a), 6(b), and $6(\mathrm{c})$, respectively.

\section{Conclusions}

Based on the above theoretical analysis and numerical calculation, the following conclusions are obtained.

In this paper, the model of two rotors coupled with a weak spring is proposed to study the synchronization characteristics by the average method of small parameters. When the values of the system parameters satisfy the equilibrium equations and the stability criteria, the vibration system will implement synchronization. The above-mentioned study indicated there are many factors having influence on the stability of the system of self-synchronization, which mainly include the spring stiffness and the stiffness of connecting spring and installation location. Additional, it can be see that the phase difference is close to $0^{\circ}$ within the limits of the stiffness by appropriately selecting the stiffness of the connecting spring, which results in both vibration amplitude and the screening efficiency of the system which could be improved. Finally, the computer simulations are used to verify the correctness of the theoretical analysis.

\section{Competing Interests}

The authors declare that there are no competing interests regarding the publication of this paper.

\section{Acknowledgments}

This study is supported by science and technology support plan of Sichuan province (2016RZ0059).

\section{References}

[1] I. I. Blekhman, Synchronization in Science and Technology, 1988.

[2] I. I. Blekhman, A. L. Fradkov, O. P. Tomchina, and D. E. Bogdanov, "Self-synchronization and controlled synchronization: general definition and example design," Mathematics and Computers in Simulation, vol. 58, no. 4-6, pp. 367-384, 2002.

[3] J. F. V. Staden and C. J. Hattingh, "Self-synchronization and controlled synchronization," in Proceedings of the 1st International Conference on Control of Oscillations and Chaos, vol. 1, pp. 3641, IEEE, St. Petersburg, Russia, August 1997.
[4] C. Huygens, Horologium Oscillatorium, Apud F. Muguet, Paris, France, 1673.

[5] P. Koluda, P. Perlikowski, K. Czolczynski, and T. Kapitaniak, "Synchronization of two self-excited double pendula," The European Physical Journal: Special Topics, vol. 223, no. 4, pp. 613-629, 2014.

[6] P. Koluda, P. Perlikowski, K. Czolczynski, and T. Kapitaniak, "Synchronization configurations of two coupled double pendula," Communications in Nonlinear Science and Numerical Simulation, vol. 19, no. 4, pp. 977-990, 2014.

[7] P. Koluda, P. Brzeski, and P. Perlikowski, "Dynamics of $n$ coupled double pendula suspended to the moving beam," International Journal of Structural Stability and Dynamics, vol. 14, no. 8, Article ID 1440028, 2014.

[8] L. Sperling, B. Ryzhik, C. Linz, and H. Duckstein, "Simulation of two-plane automatic balancing of a rigid rotor," Mathematics \& Computers in Simulation, vol. 58, no. 4-6, pp. 351-365, 2002.

[9] J. M. Balthazar, J. L. P. Felix, and R. M. L. R. F. Brasil, "Short comments on self-synchronization of two non-ideal sources supported by a flexible portal frame structure," Journal of Vibration and Control, vol. 10, no. 12, pp. 1739-1748, 2004.

[10] J. M. Balthazar, J. L. Felix, and R. M. Brasil, "Some comments on the numerical simulation of self-synchronization of four nonideal exciters," Applied Mathematics and Computation, vol. 164, no. 2, pp. 615-625, 2005.

[11] W. Bangchun, Z. Chunyu, F. Jian et al., Vibratory and Controlled Synchronization Engineering, Alpha Science International Ltd, 2012.

[12] X.-L. Zhang, B.-C. Wen, and C.-Y. Zhao, "Synchronization of three homodromy coupled exciters in a non-resonant vibrating system of plane motion," Acta Mechanica Sinica, vol. 28, no. 5, pp. 1424-1435, 2012.

[13] C.-Y. Zhao, Y.-M. Zhang, and B.-C. Wen, "Synchronisation and general dynamic symmetry of a vibrating system with two exciters rotating in opposite directions," Chinese Physics B, vol. 19, no. 3, Article ID 030301, 2010.

[14] X. Zhang, B. Wen, and C. Zhao, "Synchronization of three nonidentical coupled exciters with the same rotating directions in a far-resonant vibrating system," Journal of Sound \& Vibration, vol. 332, no. 9, pp. 2300-2317, 2013.

[15] G. S. M. Ngueuteu, R. Yamapi, and P. Woafo, "Effects of higher nonlinearity on the dynamics and synchronization of two coupled electromechanical devices," Communications in Nonlinear Science and Numerical Simulation, vol. 13, no. 7, pp. 1213-1240, 2008.

[16] M. Kumon, R. Washizaki, J. Sato, R. Kohzawa, I. Mizumoto, and Z. Iwai, "Controlled synchronization of two 1-DOF coupled oscillators," IFAC Proceedings Volumes, vol. 35, no. 1, pp. 109-114, 2002.

[17] A. L. Fradkov and B. Andrievsky, "Synchronization and phase relations in the motion of two-pendulum system," International Journal of Non-Linear Mechanics, vol. 42, no. 6, pp. 895-901, 2007.

[18] P. Fang, Y. Hou, Y. Nan, and L. Yu, "Study of synchronization for a rotor-pendulum system with Poincare method," Journal of Vibroengineering, vol. 17, no. 5, pp. 2681-2695, 2015. 


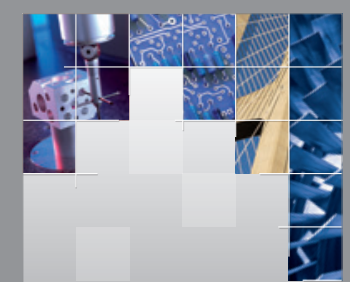

\section{Enfincering}
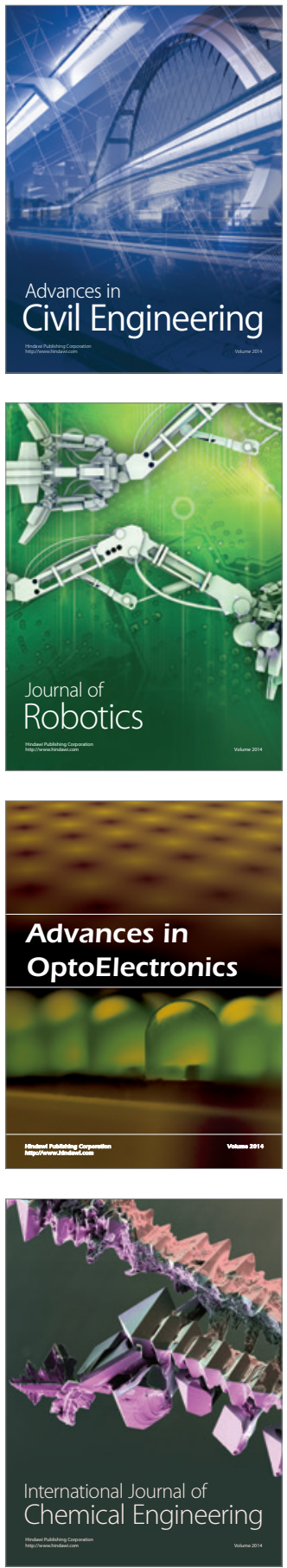

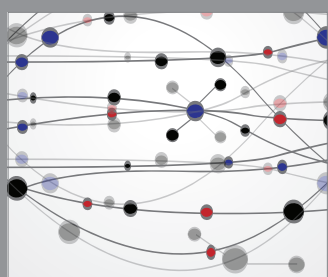

The Scientific World Journal

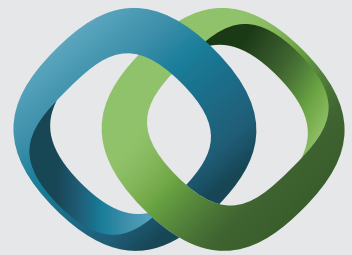

\section{Hindawi}

Submit your manuscripts at

http://www.hindawi.com
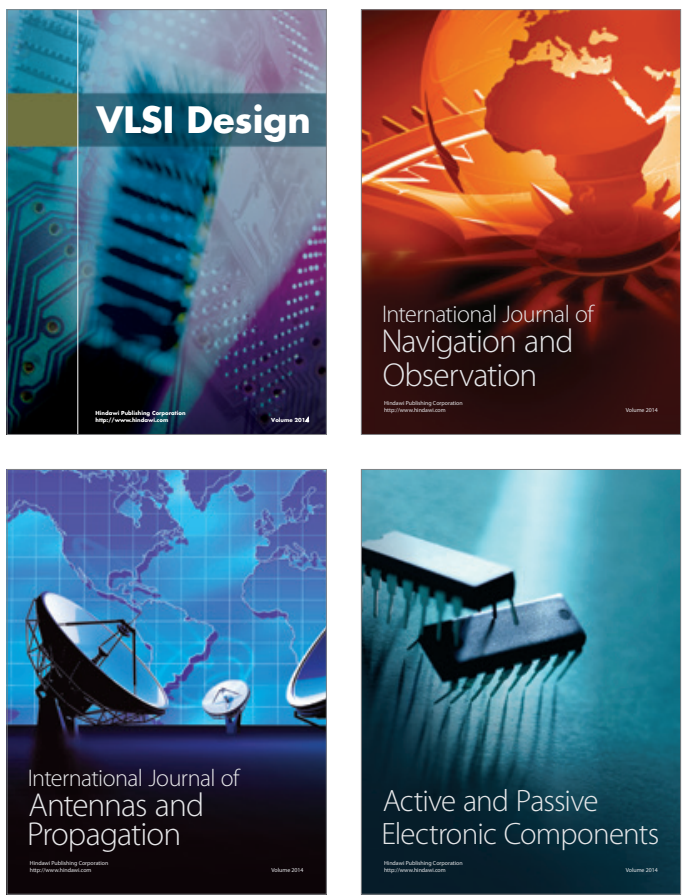
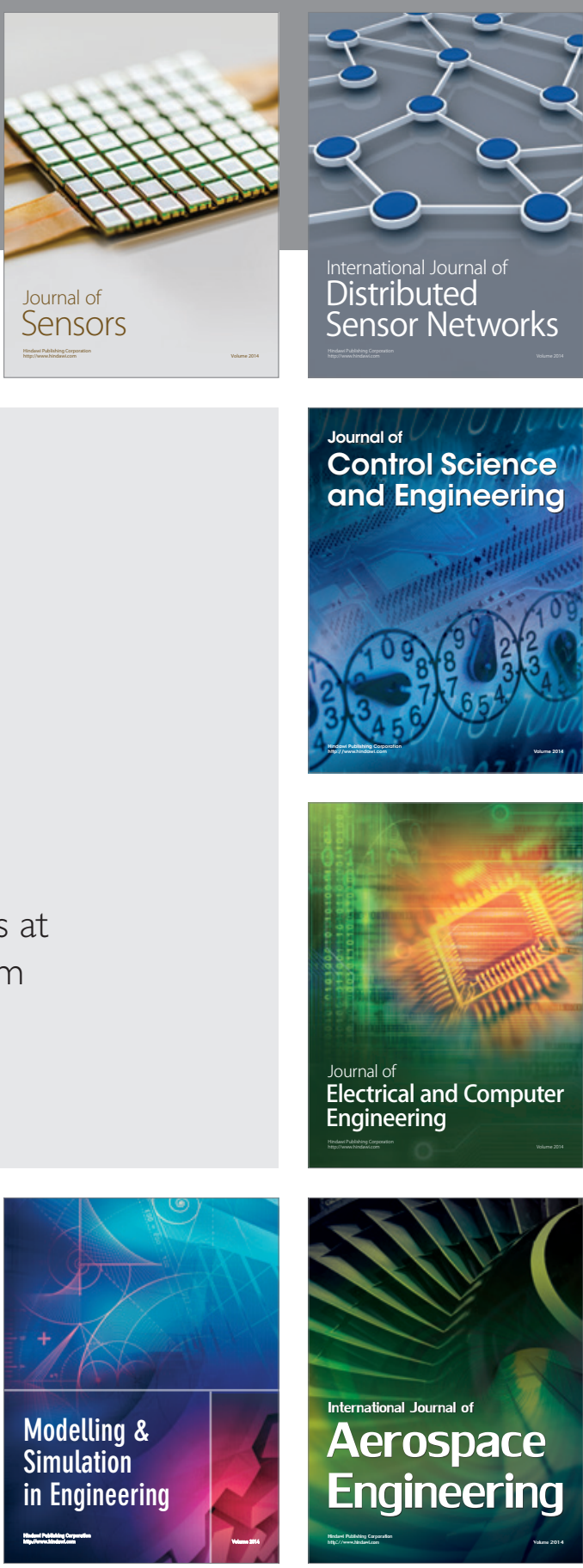

International Journal of

Distributed

Sensor Networks

Journal of

Control Science

and Engineering
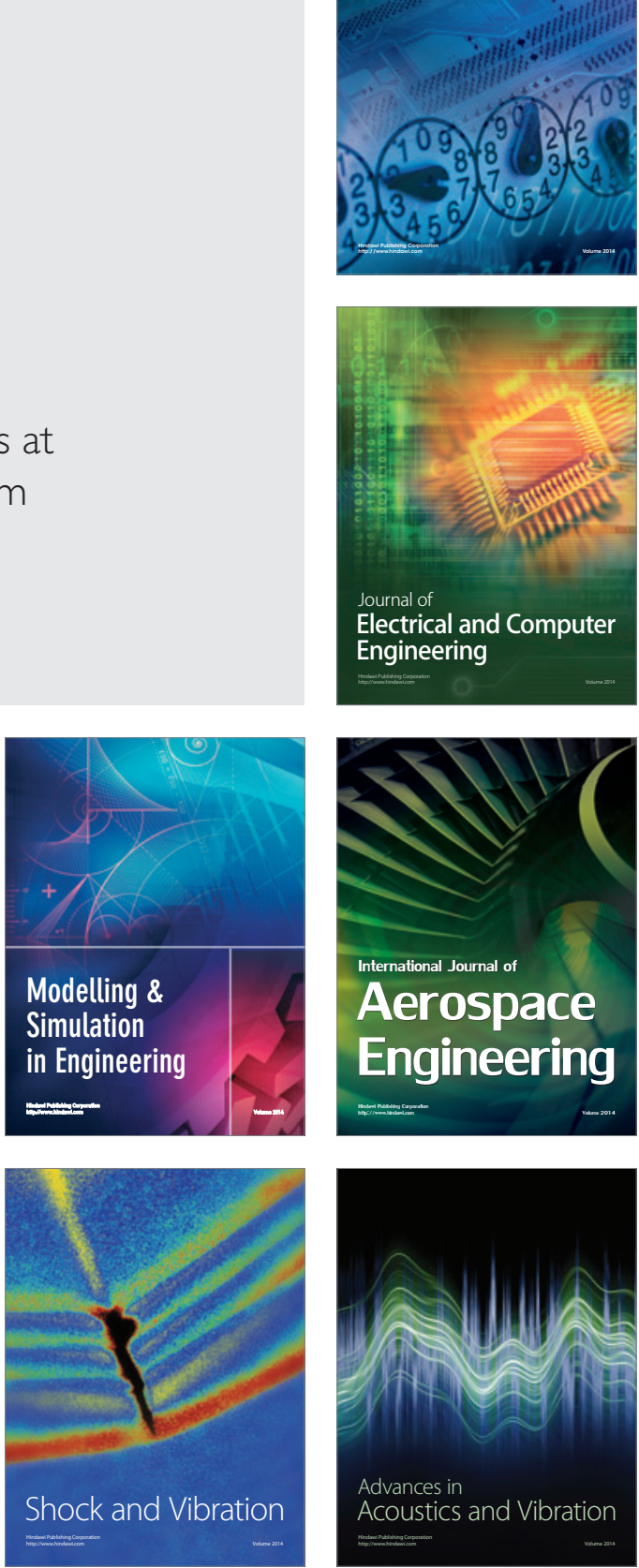